\title{
Transient Combined Convective Heat Transfer over a Stretching Surface in a Non-Newtonian Nanofluid Using Buongiorno's Model
}

\author{
Rama Subba Reddy Gorla1, Buddakkagari Vasu², Sadia Siddiqa ${ }^{3}$ \\ ${ }^{1}$ Department of Mechanical Engineering, University of Akron, Akron, OH, USA \\ ${ }^{2}$ Department of Mathematics, Motilal Nehru National Institute of Technology, Allahabad, India \\ ${ }^{3}$ Department of Mathematics, COMSATS Institute of Information Technology, Attock, Pakistan \\ Email: rgorla@uakron.edu, bvsmaths@gmail.com, saadiasiddiqa@gmail.com
}

Received 30 December 2015; accepted 26 February 2016; published 29 February 2016

Copyright (C 2016 by authors and Scientific Research Publishing Inc.

This work is licensed under the Creative Commons Attribution International License (CC BY).

http://creativecommons.org/licenses/by/4.0/

(c) (i) Open Access

\section{Abstract}

The present paper investigates the transient mixed convective boundary layer flow of an incompressible non-Newtonian quiescent nanofluid adjacent to a vertical stretching surface. The effects of the Brownian motion and thermophoresis are included for the nanofluid. Using appropriate non-similarity transformations the non-dimensional, coupled and highly non-linear system of equations is solved numerically using the efficient Keller-box implicit finite difference method for the whole transient from $t=0$ (initial state) to $t \rightarrow \infty$ (final steady-state flow). The box method is unconditionally stable. Numerical results for dimensionless velocity $\left(f^{\prime}\right)$, micro-rotation $(g)$, temperature $(\theta)$, nanoparticle volume fraction $(\phi)$ at final steady state flow, skin friction function $\left(C_{f} R e_{x}^{1 / 2}\right)$, Nusselt number function $\left(N u_{x} / R e_{x}^{1 / 2}\right)$ and Sherwood number function $\left(S h_{x} / R e_{x}^{1 / 2}\right)$ have been presented on various parameters inform of tables and graphs. The results indicate that as $\mathrm{Nb}$ and $N t$ increase, the Nusselt number decreases whereas Sherwood number increases at initial and early state time but decreases at the final steady state time. As the $K$ increases, the friction factor decreases whereas surface mass transfer rate and the surface heat transfer rates slightly increase. The results reveal that there is a smooth transition of flow from unsteady state to the final steady state. A special case of our results is in good agreement with an earlier published work. The study has many practical applications such as extrusion of plastic sheets, paper production, glass blowing, metal spinning and drawing plastic films.

\section{Keywords}

Nanofluid, Mixed Convection, Non-Newtonian Fluid, Brownian Motion, Keller-Box Numerical 


\section{Method, Boundary Layers}

\section{Introduction}

Mixed convection heat transfer problems in the boundary layer flows adjacent to the surface have many important applications in production and manufacturing processes. Some of the practical applications involve extrusion of plastic sheets, paper production, glass blowing, metal spinning and drawing plastic films.

The efficiencies of thermal devices and systems are related to heat transfer rates which in turn depend on the thermal conductivity of the working fluids. Despite considerable previous research and development efforts onheat transfer enhancement, the demand is growing for more efficient and robust heat transfer fluids with significantly higher thermal conductivities than traditional ones. Nanofluids are engineered by suspending nanoparticles with average sizes below $100 \mathrm{~nm}$ in traditional heat transfer fluids such as water, oil, and ethylene glycol. Many interesting properties of nanofluids have been reported in the past decades. The comprehensive references on nanofluids can be found in the recent book by [1] and in the review papers by [2]-[7] etc. They present the recent developments in the study of nanofluids, including the preparation methods, the evaluation methods for their stability, the ways to enhance their stability, the stability mechanisms, and their potential applications in heat transfer intensification, mass transfer enhancement, energy fields, mechanical fields, biomedical fields, and so forth.

An analytic study on the onset of convection in a horizontal layer of a porous medium with the Brinkman model and the Darcy model filled with a nanofluid was presented by [8] [9]. The flow and heat transfer characteristics on a moving plate in a nanofluid were investigated [10]. [11] studied the mixed Convection Flow of Non-Newtonian fluid from a Slotted Vertical Surface with Uniform Surface Heat Flux. [12] investigated theoretically the mixed convection in an axisymmetric stagnation flow of a non-Newtonian nanofluid on a vertical cylinder. [13] considered the unsteady free convective boundary layer flow of a nanofluid over a vertical cylinder. [14] studied the effect of Soret parameter on mixed convective flow along a vertical plate in a nanofluid under convective boundary conditions. A clear picture about the nanofluid boundary layer flows was still to emerge. [15] had been analyzed unsteady convective flow of a nanofluid over a stretching surface with variable transport properties and radiation effect in the presence of heat source. Very recently [16], an unsteady flow, heat and mass transfer in a nanofluid over a stretching sheet was considered.

In recent years, the dynamics of micropolar fluids, originated from the theory of [17] [18] has been a popular area of search. Micropolar fluids are a special class of micromorphic fluids, in which the elements are allowed to undergo rigid rotations only without stretch. In thermo-micropolar fluid mechanics, the classical continuum and thermodynamics laws are extended with additional equations which account for the conservation of micro-inertia moments and the balance of first stress moments which arise due to the consideration of microstructure in a fluid. Hence, new kinematic variables (gyration tensor, micro inertia moment tensor), and concepts of body moments, stress moments and microstress are combined with classical continuum fluid dynamics theory. Thermo-micropolar fluids can accurately simulate liquids consisting of randomly oriented particles suspended in a viscous medium and offer an excellent framework to study advanced chemical engineering flow regimes. The theory may be applied to explain the flow of colloidal solutions, liquid crystals, fluids with additives, suspension solutions, blood flows, fluid with bar like elements, etc. An excellent early review about micropolar fluid mechanics is provided by [19] [20]. Thermal convection flows of micropolar fluids have been studied intensely by using numerical methodologies by [21]-[24]. [25] studied mixed convection micropolar boundary layers on a permeable sphere by Mangler transformation and potential outer flow velocity. [26] also studied mixed convection boundary layer flow about an isothermal sphere in micropolar fluid.

Moreover, combined heat and mass transfer problems over stretching surface is important in extrusion processes. The production of sheeting material arises in a number of industrial manufacturing processes and includes both metal and polymer sheets. The quality of the final product depends on the rate of heat transfer at the stretching surface. The boundary layer flow due to a stretching vertical surface in a quiescent viscous and incompressible fluid when the buoyancy forces are taken into account have been considered only with steady-state flow, but the flow and thermal fields may be unsteady due to either impulsive stretching of the surface or external stream and sudden changes in the surface temperature. In many other engineering applications, unsteadiness 
is an integral part of the problem. [27] studied the unsteady free convection flow over a continuous moving vertical surface in an ambient fluid, and [28] [29] investigated theoretically various stretching problems in micropolar fluids. Further, [30] [31] deal with the unsteady boundary layer flow due to impulsive starting from rest of a stretching sheet in a viscous fluid. [32] studied the coupled heat and mass transfer intransient flow by mixed convection past a vertical stretching sheet embedded in a fluid-saturated porous medium in the presence of a chemical reaction effect. [33] considered the flow and heat transfer over an unsteady shrinking sheet with suction in nanofluids.

Motivated by the above-mentioned investigations and applications, in this present paper the main objective is to investigate the unsteady mixed convective boundary layer flow of an incompressible non-Newtonian nanofluid over a stretching vertical surface in a quiescent viscous and incompressible fluid. The micropolar model is chosen for the non-Newtonian fluid since the spinning motion of the nanoparticles as they move along the stream-wise direction can be best described by the micropolar fluid model. The effects of the Brownian motion and thermophoresis are included for the nanofluid. The effects of the governing multi-physical parameters on heat and mass transfer characteristics are analyzed using a robust, implicit, well-tested finite difference method for the whole transient from $t=0$ (initial state) to $t \rightarrow \infty$ (final steady-state flow). Such a study has to the authors' knowledge thus far not appeared in the scientific literature.

\section{Mathematical Model}

Consider an unsteady, two dimensional, incompressible, combined convective boundary-layer flow of a micropolar nanofluid in the region $y>0$ driven by a plane surface located at $y=0$ with a fixed end at $x=0$. The micropolar fluid model is selected to describe the motion of the solid nano particles. It is assumed the velocity components in $x$ and $y$ directions are $u$ and $v$, respectively. We assume that $T_{w}$ and $C_{w}$ are the temperature and species (nanoparticle) concentration at the wall and $T_{\infty}, C_{\infty}$ are the temperature and species concentration far away from the sheet, respectively. The $\mathrm{x}$-axis is taken along the stretching direction of the surface with the stretching rate $U_{e}(x)=a x$ where $a>0$, and y-axis is perpendicular to it. When considering micro/nanoscale fluid flows several effects become increasingly important, which are typically excluded from the macroscale fluid flow. The non-Newtonian nanofluid model incorporates the effects of Brownian motion and thermophoresis. It is also assumed that all the fluid properties are constant except that of the influence of the density variation with temperature and the nanoparticles volume faction in the body force term (Boussinesq's approximation). Figure 1 shows the schematic coordinate system and flow model.

Under the usual boundary layer approximation, the conservation equations for mass, momentum, energy and concentration can be shown to take the form:

Conservation of mass:

$$
\frac{\partial u}{\partial x}+\frac{\partial v}{\partial y}=0
$$

Conservation of linear momentum:

$$
\frac{\partial u}{\partial t}+u \frac{\partial u}{\partial x}+v \frac{\partial u}{\partial y}=\left(v+\frac{\kappa}{\rho}\right) \frac{\partial^{2} u}{\partial y^{2}}+\frac{\kappa}{\rho} \frac{\partial N}{\partial y}+\left[\left(1-C_{\infty}\right) \rho_{f \infty} g \beta\left(T-T_{\infty}\right)-\left(\rho_{p}-\rho_{f \infty}\right) g\left(C-C_{\infty}\right)\right]
$$

Conservation of angular momentum:

$$
\rho j\left(\frac{\partial N}{\partial t}+u \frac{\partial N}{\partial x}+v \frac{\partial N}{\partial y}\right)=-\kappa\left(2 N+\frac{\partial u}{\partial y}\right)+\gamma \frac{\partial^{2} N}{\partial y^{2}}
$$

Conservation of energy:

$$
\frac{\partial T}{\partial t}+u \frac{\partial T}{\partial x}+v \frac{\partial T}{\partial y}=\alpha \frac{\partial^{2} T}{\partial y^{2}}+\tau\left[D_{B} \frac{\partial C}{\partial y} \frac{\partial T}{\partial y}+\frac{D_{T}}{T_{\infty}}\left(\frac{\partial T}{\partial y}\right)^{2}\right]
$$

Conservation of nanoparticle volume-fraction:

$$
\frac{\partial C}{\partial t}+u \frac{\partial C}{\partial x}+v \frac{\partial C}{\partial y}=D_{B} \frac{\partial^{2} C}{\partial y^{2}}+\frac{D_{T}}{T_{\infty}} \frac{\partial^{2} T}{\partial y^{2}}
$$




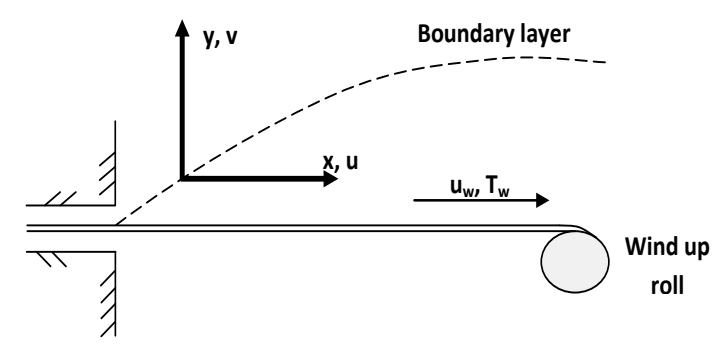

(a)

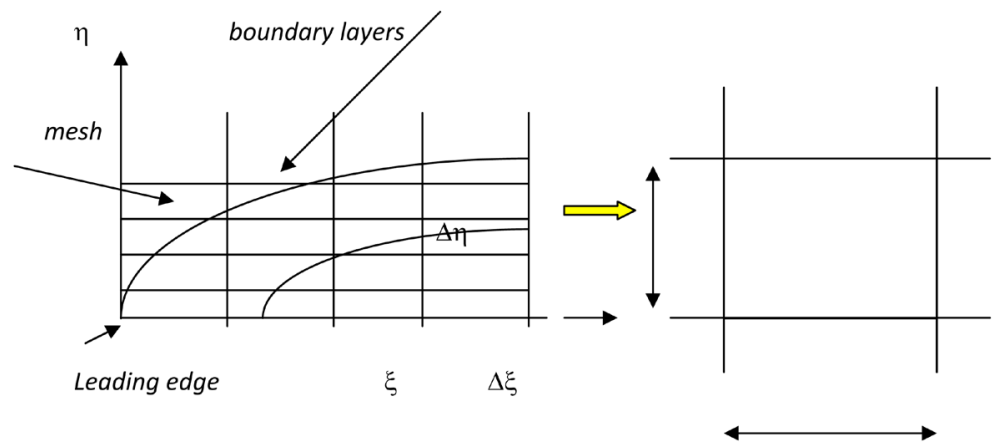

(b)

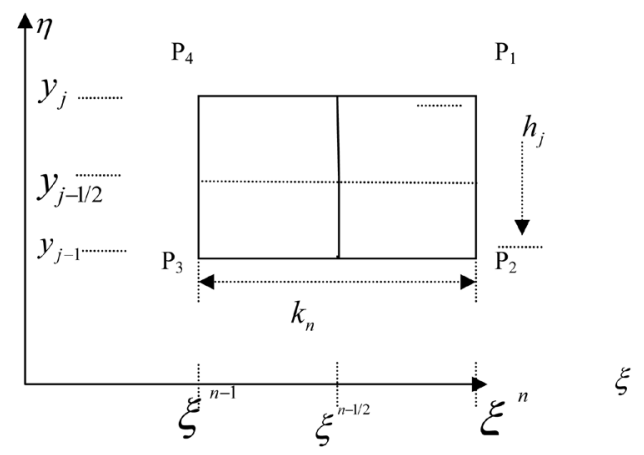

(c)

Figure 1. (a) Physical model and coordinate system; (b) Grid meshing and a Keller Box computational cell; (c) Net “Keller-Box” for difference approximations.

where $\alpha=\frac{k}{(\rho C)_{f}}, \tau=\frac{(\rho C)_{p}}{(\rho C)_{f}}, \gamma=(\mu+(\kappa / 2)) j$ is the gyro-viscosity of micropolar fluid, $j$ is the micro-inertia per unit, $g$ is the acceleration due to gravity, $\rho$ is the fluid density, $\kappa$ is the thermal conductivity, $\mu$ is the viscosity of the fluid, $T$ and $C$ are the temperature and concentration, $\rho_{f}$ is the density of the base fluid and $\rho_{P}$ is the density of the nanoparticles. $D_{B}$ and $D_{T}$ are the Brownian diffusion coefficient and the thermophoresis is diffusion coefficient, respectively, $(\rho C)_{f}$ and $(\rho C)_{P}$ are the heat capacity of the base fluid and the effective heat capacity of the nanoparticles material, respectively. $\alpha$ is the thermal diffusivity.

The boundary conditions appropriate for the regime corresponding to Equations (1)-(5) are:

$$
\begin{gathered}
\alpha t<0, \quad u=v=0, N=0, T=T_{\infty}, C=C_{\infty} \quad \forall x, y \\
t \geq 0, \quad u=U_{e}(x), v=0, N=-n \frac{\partial u}{\partial y}, T=T_{w}, C=C_{w} \text { at } y=0 \\
u \rightarrow 0, T \rightarrow T_{\infty}, N \rightarrow 0, C \rightarrow C_{\infty} \text { as } y \rightarrow \infty
\end{gathered}
$$


The above Equations (1) to (6) can be further non-dimensionalized using new variables:

$$
\begin{gathered}
\xi=1-\mathrm{e}^{-t},, \quad \eta=\xi^{1 / 4}(1 / v)^{1 / 2} y, \psi=(v)^{1 / 2} \xi^{1 / 2} x f(\xi, \eta) \\
N=(1 / v)^{1 / 2} \xi^{-1 / 2} x g(\xi, \eta), \theta(\xi, \eta)=\frac{T-T_{\infty}}{T_{w}-T_{\infty}}, \phi(\xi, \eta)=\frac{C-C_{\infty}}{C_{w}-C_{\infty}}
\end{gathered}
$$

where $\psi$ is the stream function defined in the usual way as $u=\partial \psi / \partial y$ and $v=-\partial \psi / \partial x$. Substituting (7) into Equations (1)-(6) and after some algebraic manipulation we arrive at the following transformed equations:

$$
\begin{gathered}
(1+K) f^{\prime \prime \prime}+(1-\xi)(\eta / 2) f^{\prime \prime}+\xi\left(f f^{\prime \prime}-\left(f^{\prime}\right)^{2}\right)+\lambda \xi\left(\theta-N_{r} \phi\right)+K g^{\prime}=\xi(1-\xi) f_{\xi}^{\prime} \\
(1+K / 2) g^{\prime \prime}+(1-\xi)\left(g / 2+(\eta / 2) g^{\prime}\right)+\xi\left(f g^{\prime}-g f^{\prime}\right)-K\left(2 g+f^{\prime \prime}\right)=\xi(1-\xi) g_{\xi} \\
(1 / P r) \theta^{\prime \prime}+N_{b} \phi^{\prime} \theta^{\prime}+\xi\left(f \theta^{\prime}-\theta f^{\prime}\right)+(1-\xi)(\eta / 2) \theta^{\prime}+N_{t}\left(\theta^{\prime}\right)^{2}=\xi(1-\xi) \theta_{\xi} \\
(1 / S c) \phi^{\prime \prime}+(1-\xi)(\eta / 2) \phi^{\prime}+\xi\left(f \phi^{\prime}-\phi f^{\prime}\right)+(1 / S c)\left(N_{t} / N_{b}\right) \theta^{\prime \prime}=\xi(1-\xi) \phi_{\xi}
\end{gathered}
$$

In the above equations, the primes denote the differentiation with respect to $\eta$, the dimensionless radial coordinate $\xi, f_{\xi}^{\prime}, g_{\xi}, \theta_{\xi}, \phi_{\xi}$ are partial derivatives with respect to $\xi$, the material parameter $K$ is defined as $K=\kappa / \mu . \operatorname{Pr}=v / \alpha$ is the Prandtl number, $S c=\frac{v}{D_{B}}$ is the Schmidt number, $N r=\frac{\left(\rho_{p}-\rho_{f \infty}\right)\left(C_{w}-C_{\infty}\right)}{\rho_{f \infty} \beta\left(T_{w}-T_{\infty}\right)\left(1-C_{\infty}\right)}$ is the buoyancy ratio parameter, $N_{b}=\frac{\tau D_{B}\left(C_{w}-C_{\infty}\right)}{v}$ is the Brownian motion parameter, $N_{t}=\frac{\tau D_{T}\left(T_{w}-T_{\infty}\right)}{v T_{\infty}}$ is the thermophoresis parameter, $\lambda=\frac{G r_{x}}{R e_{x}^{2}}$ is the mixed convection parameter, $G r_{x}=\frac{\left(1-C_{\infty}\right) \rho_{f_{\infty}} g \beta\left(T_{w}-T_{\infty}\right) x^{3}}{v^{2}}$ is the local Grashof number, $R_{x}=\frac{U_{x}(x) x}{v}$ is the local Reynolds number. It may be noted that $\lambda>0$ corresponds to the assisting flow (heated plate), $\lambda<0$ corresponds to the opposing flow (cooled plate), and $\lambda=0$ corresponds to the forced convection flow, respectively.

The corresponding boundary conditions are:

$$
\begin{gathered}
f=0, f^{\prime}=1, \theta=1, g=-n f^{\prime \prime}, \phi=1 \text { at } \eta=0 \\
f^{\prime} \rightarrow 0, \theta \rightarrow 0, g \rightarrow 0, \phi \rightarrow 0 \text { as } \eta \rightarrow \infty
\end{gathered}
$$

The engineering design quantities of physical interest include the skin-friction coefficient, Nusselt number and Sherwood number, which are given by:

$$
\begin{gathered}
C_{f} \operatorname{Re}_{x}^{1 / 2}=\xi^{-1 / 2}[1+(1-n) K] f^{\prime \prime}(\xi, 0) \\
N u_{x} / \operatorname{Re}_{x}^{1 / 2}=-\xi^{-1 / 2} \theta^{\prime}(\xi, 0) \\
S h_{x} / \operatorname{Re}_{x}^{1 / 2}=-\xi^{-1 / 2} \phi^{\prime}(\xi, 0)
\end{gathered}
$$

\section{Special Cases}

To provide some means of interesting numerical calculations, we shall now obtain some special cases of the problem.

\subsection{Initial State and the Early Unsteady Flow $(0<t \ll 1)$}

For early unsteady flow $0<t \ll 1$, we have $\xi \approx 0$ so Equations (8) to (11) reduce in the leading order approximation to 


$$
\begin{gathered}
(1+K) f^{\prime \prime \prime}+(\eta / 2) f^{\prime \prime}+K g^{\prime}=0 \\
(1+K / 2) g^{\prime \prime}+\left(g / 2+(\eta / 2) g^{\prime}\right)-K\left(2 g+f^{\prime \prime}\right)=0 \\
(1 / P r) \theta^{\prime \prime}+N_{b} \phi^{\prime} \theta^{\prime}+(\eta / 2) \theta^{\prime}+N_{t}\left(\theta^{\prime}\right)^{2}=0 \\
(1 / S c) \phi^{\prime \prime}+(\eta / 2) \phi^{\prime}+(1 / S c)\left(N_{t} / N_{b}\right) \theta^{\prime \prime}=0
\end{gathered}
$$

The corresponding boundary conditions are:

$$
\begin{gathered}
f=0, \quad f^{\prime}=1, \theta=1, g=-n f^{\prime \prime}, \phi=1 \text { at } \eta=0 \\
f^{\prime} \rightarrow 0, \theta \rightarrow 0, g \rightarrow 0, \phi \rightarrow 0 \text { as } \eta \rightarrow \infty
\end{gathered}
$$

\subsection{Final Steady State Flow $(t \rightarrow \infty)$}

For this case $\xi=1$ Equations (8) to (11) takes the following forms:

$$
\begin{gathered}
(1+K) f^{\prime \prime \prime}+\left(f f^{\prime \prime}-\left(f^{\prime}\right)^{2}\right)+\lambda\left(\theta-N_{r} \phi\right)+K g^{\prime}=0 \\
(1+K / 2) g^{\prime \prime}+\left(f g^{\prime}-g f^{\prime}\right)-K\left(2 g+f^{\prime \prime}\right)=0 \\
(1 / P r) \theta^{\prime \prime}+N_{b} \phi^{\prime} \theta^{\prime}+\left(f \theta^{\prime}-\theta f^{\prime}\right)+N_{t}\left(\theta^{\prime}\right)^{2}=0 \\
(1 / S c) \phi^{\prime \prime}+\left(f \phi^{\prime}-\phi f^{\prime}\right)+(1 / S c)\left(N_{t} / N_{b}\right) \theta^{\prime \prime}=0
\end{gathered}
$$

subject to the boundary conditions (20).

\section{Numerical Solution with Implicit Finite Difference Code}

The Equations (8)-(11) governing the present problem under the boundary conditions (12) were solved numerically by the Keller-Box method described in the book by [34] which is an implicit finite difference scheme with unconditionally stable. This method, originally developed for low speed aerodynamic boundary layers by [35] has been employed in a diverse range of nonlinear magnetohydrodynamics and coupled heat transfer problems. Very few of these papers however have provided guidance for researchers as to customization of the Keller-box scheme to magnetohydrodynamic heat transfer problems. We therefore present a more detailed exposition here. Essentially 4 phases are central to the Keller Box Scheme. These are:

a) Reduction of the Nth order partial differential equation system to $\mathrm{N}$ 1st order equations

b) Finite difference discretization

c) Quasilinearization of Non-Linear Keller Algebraic Equations

d) Block-tridiagonal elimination of Linear Keller Algebraic Equations

A 2-dimensional computational grid is imposed on the $\xi-\eta$ plane as sketched Figure 1(b). The stepping process is defined by:

$$
\begin{aligned}
& \xi_{o}=0 ; \quad \xi_{n}=\xi_{n-1}+k_{n}, n=1,2, \cdots, N \\
& \eta_{0}=0 ; \eta_{j}=\eta_{j-1}+h_{j}, j=1,2, \cdots, J
\end{aligned}
$$

where $k_{n}$ and $h_{j}$ denote the step distances in the $\xi$ and $\eta$ directions respectively. Denoting $\Sigma$ as the value of any variable at station $\xi_{n}, \eta_{j}$, and the following central difference approximations are substituted for each reduced variable and their first order derivatives, viz:

$$
\begin{gathered}
(\Sigma)_{j-1 / 2}^{n-1 / 2}=\left[\Sigma_{j}^{n}+\Sigma_{j-1}^{n}+\Sigma_{j}^{n-1}+\Sigma_{j-1}^{n-1}\right] / 4 \\
(\partial \Sigma / \partial \xi)_{j-1 / 2}^{n-1 / 2}=\left[\Sigma_{j}^{n}+\Sigma_{j-1}^{n}+\Sigma_{j}^{n-1}+\Sigma_{j-1}^{n-1}\right] / 4 k_{n} \\
(\partial \Sigma / \partial \eta)_{j-1 / 2}^{n-1 / 2}=\left[\Sigma_{j}^{n}+\Sigma_{j-1}^{n}+\Sigma_{j}^{n-1}+\Sigma_{j-1}^{n-1}\right] / 4 h_{j}
\end{gathered}
$$

where $k_{n}=$ streamwise stepping distance ( $\xi$-mesh spacing) and $h_{j}=$ spanwise stepping distance ( $\eta$-mesh spacing) defined as follows: 


$$
\begin{aligned}
& \eta_{j-1 / 2}=\left[\eta_{j}+\eta_{j-1}\right] / 2 \\
& \xi^{n-1 / 2}=\left[\xi^{n}+\xi^{n-1}\right] / 2 .
\end{aligned}
$$

\section{Phase a) Reduction of the Nth order partial differential equation system to $N$ 1st order equations}

Equations (15) to (18) subject to the boundary conditions (19) are first written as a system of first-order equations. For this purpose, we introduce new dependent variables $u(\xi, \eta), v(\xi, \eta), p(\xi, \eta), t(\xi, \eta)$ and $m(\xi, \eta)$, and $g(\xi, \eta), s=\theta(\xi, \eta), z=\varphi(\xi, \eta)$ as the variables for temperature, concentration respectively. Therefore, we obtain the following nine first-order equations:

$$
\begin{aligned}
& f^{\prime}=u \\
& u^{\prime}=v \\
& g^{\prime}=p \\
& s^{\prime}=t \\
& z^{\prime}=m \\
&(1+K) v^{\prime}+(1-\xi) \frac{\eta}{2} v+\xi\left(f v-u^{2}\right)+K p+\lambda \xi(s-N r z)=\xi(1-\xi) \frac{\partial u}{\partial \xi} \\
&(1+K / 2) p^{\prime}+\frac{1}{2}(1-\xi)[g+\eta p]+\xi(f p-g u)+K \xi(2 g+v)=\xi(1-\xi) \frac{\partial g}{\partial \xi} \\
& \frac{t^{\prime}}{P r}+(1-\xi) \frac{\eta}{2} t+\xi(f t-u s)+N b t m+N t t^{2}=\xi(1-\xi) \frac{\partial s}{\partial \xi} \\
& \frac{m^{\prime}}{S c}+(1-\xi) \frac{\eta}{2} m+\xi(f m-u z)+\frac{1}{S c} \frac{N t}{N b} t^{\prime}=\xi(1-\xi) \frac{\partial z}{\partial \xi}
\end{aligned}
$$

where primes denote differentiation with respect to $y$. In terms of the dependent variables, the boundary conditions become:

$$
\begin{aligned}
& y=0: \quad u=1, \quad f=0, \quad s=1, \quad g=-n v, \quad z=1 \\
& y \rightarrow \infty: \quad u \rightarrow 0, \quad s \rightarrow 0, \quad g \rightarrow 0, \quad z \rightarrow 0 .
\end{aligned}
$$

\section{Phase b) Finite difference discretization}

The net rectangle considered in the $\xi$ - $\eta$ plane is shown in Figure 1(c), and the net points are denoted by:

$$
\begin{gathered}
\xi^{0}=0, \quad \xi^{n}=\xi^{n-1}+k_{n}, \quad n=1,2, \cdots, N \\
\eta_{0}=0, \quad \eta_{j}=\eta_{j-1}+h_{j}, \quad j=1,2, \cdots, J, \quad \eta_{J} \equiv \eta_{\infty},
\end{gathered}
$$

where $k_{n}$ is the $\Delta \xi$-spacing and $h_{j}$ is the $\Delta \eta$-spacing. Here $n$ and $j$ are just sequence numbers that indicate the coordinate location. We approximate the quantities $(f, u, v, g, p, s, t)$ at points $\left(\xi^{n}, \eta_{j}\right)$ of the net by $\left(f_{j}^{n}, u_{j}^{n}, v_{j}^{n}, g_{j}^{n}, p_{j}^{n}, s_{j}^{n}, t_{j}^{n}\right)$, which we denote as net functions. We also employ the notion ()$_{j}^{n}$ for points and quantities midway between net points and for any net function:

$$
\begin{array}{cc}
\xi^{n-1 / 2} \equiv \frac{1}{2}\left(\xi^{n}+\xi^{n-1}\right), & \eta_{j-1 / 2} \equiv \frac{1}{2}\left(\eta_{j}+\eta_{j-1}\right) \\
()_{j}^{n-1 / 2}=\frac{1}{2}\left[()_{j}^{n}+()_{j}^{n-1}\right] \text { and } \quad()_{j-1 / 2}^{n}=\frac{1}{2}\left[()_{j}^{n}+()_{j-1}^{n}\right]
\end{array}
$$

The derivatives in the $x$-direction are replaced by finite difference approximations. For any net function ( ), generally we have:

$$
\frac{\partial()}{\partial x}=\frac{()^{n}-()^{n-1}}{k_{n}}
$$


Further details of the solution procedure are documented in for example [36], and omitted here for conservation of space. Effectively the complete linearized system is formulated as a block matrix system where each element in the coefficient matrix is a matrix itself. The numerical results are affected by the number of mesh points in both directions. Accurate results are produced by performing a mesh sensitivity analysis. After some trials in the $y$-direction a larger number of mesh points are selected whereas in the $x$-direction significantly less mesh points are utilized. $y_{\max }$ has been set at 10 and this defines an adequately large value at which the prescribed boundary conditions are satisfied. $x_{\max }$ is set at 1.0 for this flow domain. We have used 101 grid points in the $\xi$-direction and 501 grid points in the $\eta$-direction. The uniform step sizes 0.01 in $\xi$ and 0.02 in $\eta$-directions respectively, are employed. To ensure grid independence a mesh sensitivity exercise has been performed. The convergence criterion has been set at $10^{-5}$ as the difference between the current and previous iterations for the desired accuracy.

\section{Results and Discussion}

A detailed parametric study has been performed for the influence of Material parameter (K), buoyancy parameter $(\lambda)$, Brownian motion parameter $(N b)$, buoyancy ratio parameter $(N r)$, Thermophoretic parameter $(N t)$, Prandtl number $(\mathrm{Pr})$, and Schmidt number $(S c)$ on dimensionless velocity $\left(f^{\prime}\right)$, micro-rotation $(g)$, temperature $(\theta)$, nanoparticle volume fraction $(\phi)$ at final steady state, skin friction function $\left(C_{f} R e_{x}^{1 / 2}\right)$, Nusselt number function $\left(N u_{x} / R e_{x}^{1 / 2}\right)$ and Sherwood number function $\left(S h_{x} / R e_{x}^{1 / 2}\right)$. In order to assess the accuracy of the numerical solution, we tabulated results for local Skin friction and Nusselt number for different values of $\lambda$ and $\operatorname{Pr}$ when $N b=N r=N t=0, K=0, n=0, S c=0.0$ at the final steady state flow $(\xi=1)$. A comparison of the present results with literature data as reported by [28] suggests the present results are excellent coincide. Therefore, we believe that the comparison supports very well validity of the present results.

The viscous boundary layer flow develops slowly over the stretching surface and it becomes a fully developed flow after some time. The development of the boundary layer takes place in two stages. For small time $(t \ll 1$, i.e. $0<\xi \ll 1$ ), the flow is dominated by the viscous forces and the convective acceleration plays only a minor role in the flow development, the flow is unsteady. For large time $(t \rightarrow \infty$, i.e. $\xi=1)$, the flow is dominated by the viscous forces and the convective acceleration, and the unsteady acceleration plays a minorrole in the flow development, the flow becomes steady. For the intermediate region, $1<t<\infty$, i.e. $0<\xi<1$ ), there is a smooth transition from unsteady to steady flow or the transition from the unsteady to steady flow takes place without a singularity or flow instability (or formation of cells). All the obtained results furnished in Tables 1-6 for Skin friction, Nusselt and Sherwood coefficients values when final steady-state as well as unsteady flows and figures 2 to 8 for various flow distributions for different thermophysical parameters.

Table 1. Comparison of values of Skin friction $\left(C_{f} R e_{x}^{1 / 2}\right)$ and Nusselt $\left(N u_{x} / R e_{x}^{1 / 2}\right)$ coefficients values when $\xi=1$ (final steady-state flow) for various values of $\lambda$ and $\operatorname{Pr}$ when $\mathrm{Nb}=\mathrm{Nr}=\mathrm{Nt}=0, \mathrm{~K}=0, \mathrm{n}=0$, Sc $=0.0$.

\begin{tabular}{|c|c|c|c|c|c|c|c|c|}
\hline \multirow[b]{2}{*}{$\operatorname{Pr} l \lambda$} & \multicolumn{4}{|c|}{$C_{f} R e_{x}^{1 / 2}$} & \multicolumn{4}{|c|}{$N u_{x} / R e_{x}^{1 / 2}$} \\
\hline & -1 & 0 & 1 & 10 & -1 & 0 & 1 & 10 \\
\hline 0.01 & - & $\begin{array}{l}-1.0000 \\
(-1.000)\end{array}$ & $\begin{array}{c}-0.0914 \\
(-0.1927)\end{array}$ & $\begin{array}{c}4.7142 \\
(4.7130)\end{array}$ & - & $\begin{array}{c}0.0199 \\
(0.0198)\end{array}$ & $\begin{array}{c}0.1009 \\
(0.1002)\end{array}$ & $\begin{array}{c}0.1698 \\
(0.1696)\end{array}$ \\
\hline 0.7 & - & $\begin{array}{l}-1.0000 \\
(-1.000)\end{array}$ & $\begin{array}{c}-0.5046 \\
(-0.5076)\end{array}$ & $\begin{array}{c}2.5786 \\
(2.5777)\end{array}$ & - & $\begin{array}{c}0.7947 \\
(0.7937)\end{array}$ & $\begin{array}{c}0.8962 \\
(0.8961)\end{array}$ & $\begin{array}{c}1.1727 \\
(1.1724)\end{array}$ \\
\hline 1 & - & $\begin{array}{l}-1.0000 \\
(-1.000)\end{array}$ & $\begin{array}{c}-0.5610 \\
(-0.5608)\end{array}$ & $\begin{array}{c}2.3067 \\
(2.3041)\end{array}$ & - & $\begin{array}{c}1.0000 \\
(1.0000)\end{array}$ & $\begin{array}{c}1.0875 \\
(1.0873)\end{array}$ & $\begin{array}{c}1.3720 \\
(1.3716)\end{array}$ \\
\hline 3 & $\begin{array}{c}-1.3162 \\
(-1.3188)\end{array}$ & $\begin{array}{l}-1.0000 \\
(-1.000)\end{array}$ & $\begin{array}{c}-0.7106 \\
(-0.7092)\end{array}$ & $\begin{array}{c}1.4567 \\
(1.4569)\end{array}$ & $\begin{array}{c}1.8594 \\
(1.8586)\end{array}$ & $\begin{array}{c}1.9239 \\
(1.9237)\end{array}$ & $\begin{array}{c}1.9746 \\
(1.9743)\end{array}$ & $\begin{array}{c}2.2445 \\
(2.2442)\end{array}$ \\
\hline 7 & $\begin{array}{c}-1.2096 \\
(-1.2106)\end{array}$ & $\begin{array}{l}-1.0000 \\
(-1.000)\end{array}$ & $\begin{array}{c}-0.7975 \\
(-0.7962)\end{array}$ & $\begin{array}{c}0.8515 \\
(0.8505)\end{array}$ & $\begin{array}{c}3.0369 \\
(3.0361)\end{array}$ & $\begin{array}{c}3.0727 \\
(3.0722)\end{array}$ & $\begin{array}{c}3.1060 \\
(3.1055)\end{array}$ & $\begin{array}{c}3.3321 \\
(3.3318)\end{array}$ \\
\hline 10 & $\begin{array}{c}-1.1767 \\
(-1.1783)\end{array}$ & $\begin{array}{l}-1.0000 \\
(-1.000)\end{array}$ & $\begin{array}{c}-0.8264 \\
(-0.8257)\end{array}$ & $\begin{array}{c}0.6198 \\
(0.6197)\end{array}$ & $\begin{array}{c}3.6914 \\
(3.6912)\end{array}$ & $\begin{array}{c}3.7208 \\
(3.7207)\end{array}$ & $\begin{array}{c}3.7489 \\
(3.7489)\end{array}$ & $\begin{array}{c}3.9526 \\
(3.9524)\end{array}$ \\
\hline 100 & $\begin{array}{c}-1.0595 \\
(-1.0601)\end{array}$ & $\begin{array}{l}-1.0000 \\
(-1.000)\end{array}$ & $\begin{array}{c}-0.9400 \\
(-0.9400)\end{array}$ & $\begin{array}{c}-0.4064 \\
(-0.4057)\end{array}$ & $\begin{array}{c}12.2864 \\
(12.2851)\end{array}$ & $\begin{array}{c}12.2941 \\
(12.2945)\end{array}$ & $\begin{array}{c}12.3035 \\
(12.3031)\end{array}$ & $\begin{array}{c}12.3826 \\
(12.3821)\end{array}$ \\
\hline
\end{tabular}

Results in parenthesis are reported by [28]. 
Table 2. Skin friction $\left(C_{f} R e_{x}^{1 / 2}\right)$, Nusselt $\left(N u_{x} / R e_{x}^{1 / 2}\right)$ and Sherwood $\left(S h_{x} / R e_{x}^{1 / 2}\right)$ coefficients values when $\xi=1$ (final steady-state flow) for various values of $K$ and $n$ when $\operatorname{Pr}=100, \lambda=1.0, \mathrm{Nb}=\mathrm{Nr}=\mathrm{Nt}=0.1, \mathrm{Sc}=7.0$ and $\eta=7.0$.

\begin{tabular}{ccccccc}
\hline & \multicolumn{3}{c}{$C_{f} R e_{x}^{1 / 2}$} & \multicolumn{2}{c}{$N u_{x} / R e_{x}^{1 / 2}$} & \multicolumn{2}{c}{$S h_{x} / R e_{x}^{1 / 2}$} \\
\hline$K$ & $n=0$ & $n=1 / 2$ & $n=0$ & $n=1 / 2$ & $n=0$ & $n=1 / 2$ \\
\hline 0 & 2.3174 & 2.3174 & 2.1026 & 2.1026 & 3.0103 & 3.0103 \\
1 & 2.2176 & 1.4281 & 2.1021 & 2.1193 & 2.9163 & 2.8470 \\
2 & 2.0849 & 0.9306 & 2.1015 & 2.1274 & 2.8846 & 2.7809 \\
4 & 1.8159 & 0.1855 & 2.0997 & 2.1346 & 2.8633 & 2.7239 \\
5 & 1.6873 & 0.1430 & 2.0987 & 2.1364 & 2.8596 & 2.7094 \\
\hline
\end{tabular}

Table 3. Skin friction $\left(C_{f} R e_{x}^{1 / 2}\right)$, Nusselt $\left(N u_{x} / R e_{x}^{1 / 2}\right)$ and Sherwood $\left(S h_{x} / R e_{x}^{1 / 2}\right)$ coefficients values when $\xi=1$ (final steady-state flow) for various values of $\lambda$ and $\mathrm{n}$ when $\mathrm{Pr}=100, K=2.0, \mathrm{Nb}=\mathrm{Nr}=\mathrm{Nt}=0.1, \mathrm{Sc}=7.0$ and $\eta=7.0$.

\begin{tabular}{ccccccc}
\hline & \multicolumn{3}{c}{$C_{f} R e_{x}^{1 / 2}$} & \multicolumn{2}{c}{$N_{x} / R e_{x}^{1 / 2}$} & \multicolumn{2}{c}{$S h_{x} / R e_{x}^{1 / 2}$} \\
\hline$\lambda$ & $n=0$ & $n=1 / 2$ & $n=0$ & $n=1 / 2$ & $n=0$ & $n=1 / 2$ \\
\hline-2 & -10.2628 & -7.3528 & 2.3838 & 2.4640 & 1.6682 & 1.4279 \\
-1 & -5.6560 & -4.2609 & 2.2335 & 2.2789 & 2.2456 & 2.0885 \\
0 & -1.6265 & 1.5569 & 2.1535 & 2.1862 & 2.6105 & 2.4873 \\
1 & 2.0849 & 0.9306 & 2.1015 & 2.1274 & 2.8846 & 2.7809 \\
5 & 15.2264 & 9.7227 & 1.9890 & 2.0037 & 3.6132 & 3.5444 \\
10 & 29.6171 & 19.3363 & 1.9243 & 1.9342 & 4.1900 & 4.1382 \\
\hline
\end{tabular}

Table 4. Values of $C_{f} R e_{x}^{1 / 2}, N u_{x} / R e_{x}^{-1 / 2}$ and $S h_{x} / R e_{x}^{-1 / 2}$ for various values of $K, \xi$, Nt and Nb when $\operatorname{Pr}=100, \lambda=1.0, n$ $=0.5$, $\mathrm{Sc}=7.0$ and $\eta=7.0$.

\begin{tabular}{|c|c|c|c|c|c|c|c|c|c|c|c|}
\hline \multirow{3}{*}{$K$} & \multirow{3}{*}{$\xi$} & \multirow{3}{*}{$N t$} & \multirow{2}{*}{\multicolumn{3}{|c|}{$\begin{array}{c}C_{f} R e_{x}^{1 / 2} \\
N b\end{array}$}} & \multirow{2}{*}{\multicolumn{3}{|c|}{$\begin{array}{c}N u_{x} / R e_{x}^{1 / 2} \\
N \boldsymbol{b}\end{array}$}} & \multirow{2}{*}{\multicolumn{3}{|c|}{$\begin{array}{c}S h_{x} / R e_{x}^{1 / 2} \\
N \boldsymbol{b}\end{array}$}} \\
\hline & & & & & & & & & & & \\
\hline & & & 0.1 & 0.3 & 0.5 & 0.1 & 0.3 & 0.5 & 0.1 & 0.3 & 0.5 \\
\hline \multirow{9}{*}{0} & \multirow{3}{*}{0.01} & 0.1 & -4.3835 & -3.6186 & -3.3125 & 2.3343 & 2.9780 & 3.3764 & 19.4029 & 16.1139 & 15.8302 \\
\hline & & 0.3 & -3.7068 & -3.1407 & -2.8686 & -1.0815 & 0.0765 & 0.9255 & 30.9621 & 18.6016 & 16.8734 \\
\hline & & 0.5 & -3.2410 & -2.7675 & -2.4979 & -3.1579 & -1.6972 & -0.6239 & 44.4659 & 21.6286 & 18.0646 \\
\hline & \multirow{3}{*}{0.5} & 0.1 & 2.0561 & 3.6240 & 4.3876 & 2.0291 & 0.8003 & 0.4543 & 3.33382 & 3.9945 & 4.0499 \\
\hline & & 0.3 & 3.1618 & 4.3334 & 4.9262 & 1.3388 & 0.6917 & 0.4412 & 4.0455 & 4.4036 & 4.3360 \\
\hline & & 0.5 & 3.8660 & 4.8447 & 5.3372 & 1.0735 & 0.6295 & 0.4317 & 4.7843 & 4.7257 & 4.5552 \\
\hline & \multirow{3}{*}{1.0} & 0.1 & 2.3174 & 3.8937 & 4.6564 & 2.1026 & 0.8394 & 0.4933 & 3.0103 & 3.8274 & 3.9038 \\
\hline & & 0.3 & 3.3848 & 4.5752 & 5.1668 & 1.3652 & 0.7067 & 0.4581 & 3.6977 & 4.2368 & 4.1887 \\
\hline & & 0.5 & 4.0665 & 5.0659 & 5.5570 & 1.0795 & 0.6299 & 0.4336 & 4.4828 & 4.5739 & 4.4160 \\
\hline \multirow{3}{*}{1} & \multirow{3}{*}{0.01} & 0.1 & -7.0375 & -6.3702 & -6.0842 & 2.3342 & 2.9778 & 3.3764 & 19.4061 & 16.1145 & 15.8300 \\
\hline & & 0.3 & -6.4512 & -5.9278 & -5.6630 & -1.0817 & 0.0763 & 4.1565 & 30.9654 & 18.6015 & 16.8725 \\
\hline & & 0.5 & -6.0229 & -5.5656 & -5.2942 & -3.1583 & -1.6974 & -0.6241 & 44.4686 & 21.6277 & 18.0629 \\
\hline
\end{tabular}




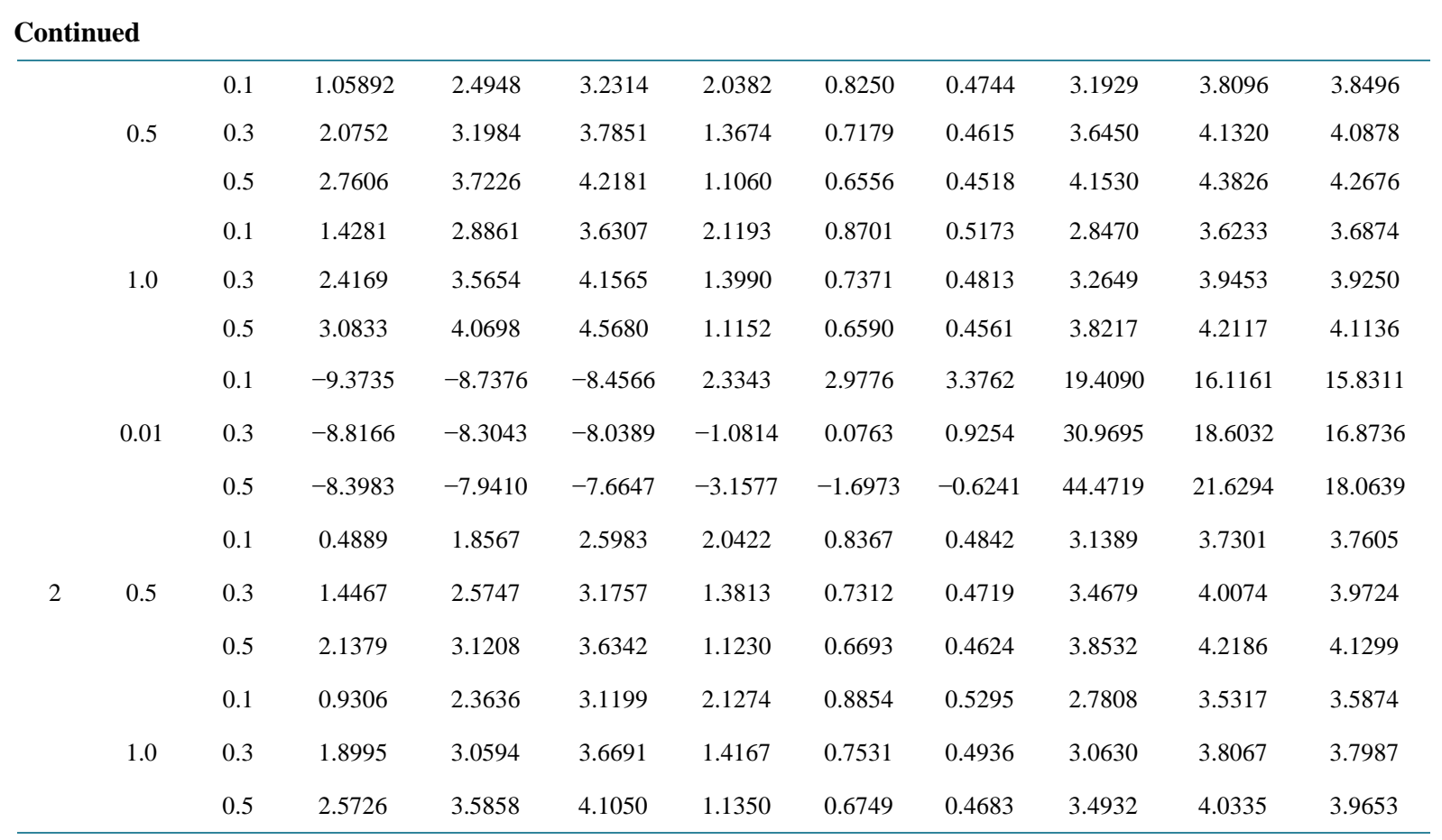

Table 5. Values of $C_{f} R e_{x}^{1 / 2}, N u_{x} / R e_{x}^{-1 / 2}$ and $S h_{x} / R e_{x}^{-1 / 2}$ for various values of $\lambda$, $\xi$, and $\operatorname{Pr}$ when $\mathrm{Nb}=\mathrm{Nr}=\mathrm{Nt}=0.1, K=$ $2.0, n=0.5$, $\mathrm{Sc}=7.0$ and $\eta=7.0$.

\begin{tabular}{|c|c|c|c|c|c|c|c|c|c|c|}
\hline \multirow{3}{*}{$\Lambda$} & \multirow{3}{*}{$\operatorname{Pr}$} & \multicolumn{3}{|c|}{$C_{f} R e_{x}^{1 / 2}$} & \multicolumn{3}{|c|}{$N u_{x} / R e_{x}^{1 / 2}$} & \multicolumn{3}{|c|}{$S h_{x} / R e_{x}^{1 / 2}$} \\
\hline & & \multicolumn{3}{|c|}{$\xi$} & \multicolumn{3}{|c|}{$\xi$} & \multicolumn{3}{|c|}{$\xi$} \\
\hline & & 0.01 & 0.5 & 1.0 & 0.01 & 0.5 & 1.0 & 0.01 & 0.5 & 1.0 \\
\hline \multirow{3}{*}{-2} & 0.7 & - & - & - & - & - & - & - & - & - \\
\hline & 7.0 & - & - & - & - & - & - & - & - & - \\
\hline & 100 & -12.3659 & -7.1777 & -7.3528 & 2.3326 & 2.1564 & 2.4640 & 19.4047 & 2.4056 & 1.4279 \\
\hline \multirow{3}{*}{-1} & 0.7 & - & - & - & - & - & - & - & - & - \\
\hline & 7.0 & - & - & - & - & - & - & - & - & - \\
\hline & 100 & -11.3681 & -4.4333 & -4.2609 & 2.3332 & 2.1088 & 2.2789 & 19.4061 & 2.6950 & 2.0885 \\
\hline \multirow{3}{*}{0} & 0.7 & -10.3707 & -1.9114 & -1.5569 & 4.4120 & 0.8815 & 0.7995 & 14.5542 & 3.1517 & 2.9199 \\
\hline & 7.0 & -10.3707 & -1.9114 & -1.5569 & 8.4141 & 2.1152 & 2.0209 & 13.2688 & 2.6002 & 2.3583 \\
\hline & 100 & -10.3707 & -1.9114 & -1.5569 & 2.3227 & 2.0708 & 2.1862 & 19.4203 & 2.9352 & 2.4873 \\
\hline \multirow{3}{*}{1} & 0.7 & -7.7489 & 4.8929 & 5.32689 & 4.4148 & 1.1696 & 1.1503 & 14.5590 & 3.6229 & 3.4891 \\
\hline & 7.0 & -9.0598 & 1.5789 & 2.1398 & 8.4150 & 2.2332 & 2.1662 & 13.2711 & 2.8346 & 2.6925 \\
\hline & 100 & -9.3735 & 0.4547 & 0.9306 & 2.3344 & 2.0422 & 2.1274 & 19.4090 & 3.1390 & 2.7809 \\
\hline \multirow{3}{*}{10} & 0.7 & 15.7508 & 41.3735 & 41.8549 & 4.4397 & 1.7728 & 1.7578 & 14.6012 & 4.8965 & 4.8078 \\
\hline & 7.0 & 2.7254 & 24.5740 & 25.4205 & 8.4274 & 2.7465 & 2.7052 & 13.2880 & 3.8019 & 3.7338 \\
\hline & 100 & -0.4021 & 18.3883 & 19.3363 & 2.3398 & 1.9127 & 1.9342 & 19.4221 & 4.2865 & 4.1382 \\
\hline \multirow{3}{*}{100} & 0.7 & 242.324 & 253.297 & 253.863 & 4.6685 & 2.9636 & 2.9516 & 14.9957 & 7.7329 & 7.6797 \\
\hline & 7.0 & 119.371 & 165.215 & 166.569 & 8.5479 & 4.0975 & 4.0719 & 13.4531 & 6.0940 & 6.0486 \\
\hline & 100 & 88.7585 & 137.807 & 139.574 & 2.3924 & 1.82744 & 1.827 & 19.5511 & 7.5423 & 7.4799 \\
\hline
\end{tabular}


Table 6. Values of $C_{f} R e_{x}^{1 / 2}, N u_{x} / R e_{x}^{-1 / 2}$ and $S h_{x} / R e_{x}^{-1 / 2}$ for various values of $\lambda, \xi$ and $\mathrm{Sc}$ when $\mathrm{Nb}=\mathrm{Nr}=\mathrm{Nt}=0.1, \mathrm{Pr}=$ $100, K=2.0, n=0.5$ and $\eta=5.0$.

\begin{tabular}{|c|c|c|c|c|c|c|c|c|c|c|}
\hline \multirow{3}{*}{$\lambda$} & \multirow{3}{*}{ Sc } & \multicolumn{3}{|c|}{$C_{f} R e_{x}^{1 / 2}$} & \multicolumn{3}{|c|}{$N u_{x} / R e_{x}^{1 / 2}$} & \multicolumn{3}{|c|}{$S h_{x} / R e_{x}^{1 / 2}$} \\
\hline & & \multicolumn{3}{|c|}{$\xi$} & \multicolumn{3}{|c|}{$\xi$} & \multicolumn{3}{|c|}{$\xi$} \\
\hline & & 0.01 & 0.5 & 1.0 & 0.01 & 0.5 & 1.0 & 0.01 & 0.5 & 1.0 \\
\hline \multirow{3}{*}{-2} & 3 & -11.7869 & -5.3447 & -5.3569 & 2.9136 & 2.9489 & 3.0893 & 13.4442 & 0.4709 & 0.0300 \\
\hline & 10 & -12.5729 & -7.8296 & -4.1241 & 3.1932 & 1.8873 & 1.9873 & 21.4453 & 3.3013 & 3.0392 \\
\hline & 50 & - & - & - & - & - & - & - & - & - \\
\hline \multirow{3}{*}{-1} & 3 & -11.0788 & -3.5817 & -3.3544 & 2.9142 & 2.9590 & 3.0813 & 13.4443 & 0.5197 & 0.1327 \\
\hline & 10 & -11.4717 & -4.7092 & -4.5860 & 3.1937 & 1.8311 & 2.0020 & 21.4475 & 3.6789 & 2.9834 \\
\hline & 50 & -11.7115 & -5.1995 & -5.2864 & 11.3467 & 0.8435 & 0.9984 & 40.0318 & 9.8861 & 8.7292 \\
\hline \multirow{3}{*}{0} & 3 & -10.3707 & -1.9114 & -1.5569 & 2.9244 & 2.9710 & 3.0923 & 13.4351 & 0.5583 & 0.1827 \\
\hline & 10 & -10.3707 & -1.9114 & -1.5569 & 3.1701 & 1.7870 & 1.8973 & 21.4912 & 3.9814 & 3.4945 \\
\hline & 50 & -10.3707 & -1.9114 & -1.5569 & 11.8997 & 0.7782 & 0.9243 & 40.2216 & 10.2971 & 9.4057 \\
\hline \multirow{3}{*}{1} & 3 & -9.6627 & -0.3212 & 0.0605 & 2.9153 & 2.9836 & 3.1234 & 13.4445 & 0.5891 & 0.1803 \\
\hline & 10 & -9.2698 & 0.6778 & 1.1641 & 3.1947 & 1.7578 & 1.8384 & 21.4518 & 4.2291 & 3.8450 \\
\hline & 50 & -9.0302 & 1.0664 & 1.5903 & 11.3456 & 0.8104 & 0.9230 & 40.0417 & 10.5838 & 9.8236 \\
\hline \multirow{3}{*}{10} & 3 & - & - & - & - & - & - & - & - & - \\
\hline & 10 & 0.6303 & 19.8895 & 20.8057 & 3.1992 & 1.6262 & 1.6537 & 21.4714 & 5.5724 & 5.3934 \\
\hline & 50 & 3.0192 & 22.5671 & 23.4130 & 11.3407 & 0.7470 & 0.8315 & 40.0862 & 12.3434 & 11.8368 \\
\hline \multirow{3}{*}{100} & 3 & - & - & - & - & - & - & - & - & - \\
\hline & 10 & 98.8822 & 145.5277 & 147.1472 & 3.2432 & 1.5290 & 1.5362 & 21.6644 & 9.2977 & 9.2075 \\
\hline & 50 & 122.0940 & 159.4513 & 160.8787 & 11.2929 & 0.6677 & 0.7256 & 40.5208 & 17.8204 & 17.5406 \\
\hline
\end{tabular}

Tables 2-6 present the values of Skin friction, Nusselt and Sherwood coefficients when final steady-state as well as unsteady cases for parametric values of variables governing the problem. Table shows that as material parameter $(K)$ increases for $n=0$ and 0.5 , the Skin friction, Nusseltand nanoparticle Sherwood coefficients decreased. This can be utilized in applications where reduced friction and heat transfer may be attractive. Table 3 shows that as buoyancy parameter $(\lambda)$ increases for $n=0$ and 0.5 , the friction factor and Sherwood number increase whereas heat transfer rate decreased. The value $n=0, N(x, 0)=0$, represents concentrated particle flows in which the particle density is sufficiently great that microelements close to the wall are unable to rotate. This case is also called strong interaction. The other case corresponding to $n=0.5$ consequences in the vanishing of antisymmetric part of the stress tensor and represents weak concentration, so that the particle suspension is fine at the boundary because of the particle rotation is equal to fluid vorticity. When $n=1$, we have flows which are representative of turbulent boundary layers. The case of $n=0.5$ is considered in the present study. Form Table 3 clearly observed the friction values are higher for $n=0$ than $n=0.5$. Also Figure 4 depicts the same trend in the flow distributions.

Table 4 shows that the friction, heat and nanoparticle mass transfer rates for various values of $K$, Nt and $\mathrm{Nb}$ at early unsteady flow and final steady flow $(\xi)$. As increase $\xi$, the friction and mass transfer rates increase whereas heat transfer rates decreased. The same trend is observed for the influence of thermophoresis parameter (Nt). Also the data interprets as increases in Brownian motion parameter (Nb), the friction and heat transfer rates are increased while nanoparticle mass transfer rates decreased.

Table 5 and Table 6 depicted the new results for small $\operatorname{Pr}=0.7, \mathrm{Sc}=3$ and large $\operatorname{Pr}=100$, Sc $=50$ values. It 
can be seen that from Table 4 that when fixed Pr, increasing $\lambda$ from a negative value to a positive value will increase friction rates, while increasing Pr leads to a decreasing of friction rates when $\lambda>0$. The reverse tendency observed when $\lambda<0$. All the friction values are coincide at a point where $\lambda=0$, i.e. when buoyancy force is zero. This is due to uncoupling of Equations (21), (22) and (23) when $\lambda=0$, in other words, the solutions to the flow field are not affected by the thermal field in which the buoyancy force is absent. Moreover, $\lambda>0$ (assisting flow) produces an increase in the friction values, while $\lambda<0$ (opposing flow) gives rise to a decrease in the local skin friction coefficient. This is because the fluid velocity increases when buoyancy force increases and hence increases the skin friction. The opposite trend occurs when buoyancy force decreases. Also, effects of $\lambda$ on the locals $k$ in friction coefficient are found to be more significant for fluids having large Prand Sc since the viscosity is greater than the fluids with small Pr and Sc. It is also observed that for particular values of Pr and Sc, the local Nusselt number is slightly increased as the buoyancy parameter $\lambda$ is increased.

Figure 2 shows that the effect of material or vortex viscosity parameter $K$ on dimensionless velocity, microrotation, temperature and nanoparticle volume fraction with $n=0.5$ for final steady-state flow $(\xi=1)$. The figure represents as increases $K$, the velocity and micro-rotation increase whereas temperature and concentration decrease. So, the velocity boundary layer thickness increases with increasing values of $K$. For a particular value of $K$, the velocity decreases monotonically with $\eta$, and tends to zero at the outside of boundary layer. This property satisfies the boundary condition $f^{\prime} \rightarrow 0$. Therefore, these figures hold the validity of the present results.

A variable relation between microrotation and the surface skin friction is appeared in the boundary condition, where $\mathrm{n}$ is constant and varies from 0 to 1 . The distributions of fully developed velocity, micro-rotation, temperature and nanoparticle volume fraction within the boundary layer for final steady-state flow $(\xi=1)$ are shown in Figure 3. Figure 3 shows that as $n$ increases, the velocity decrease but micro-rotation, temperature and nanoparticle volume fraction or concentration increase.

Figure 4 depicts the flow distributions of fully developed transient and final steady-state flow for $\operatorname{Pr}=100$ and $\lambda=1$. The large value of Prandtl number correspond the thin boundary layer; it has the more significant ap-

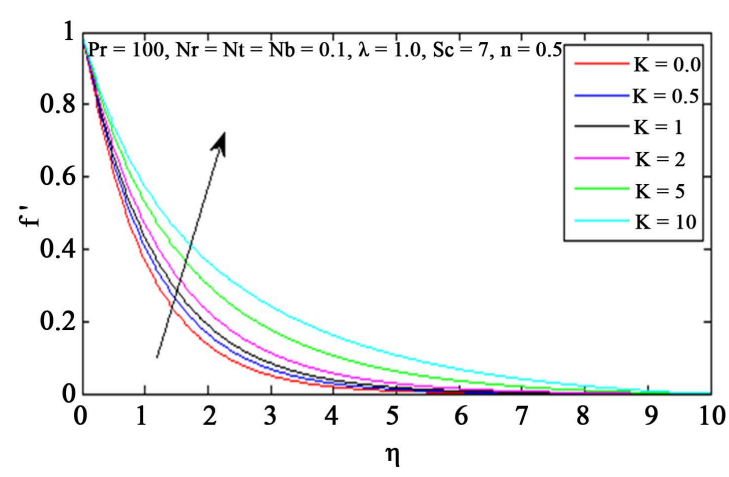

(a)

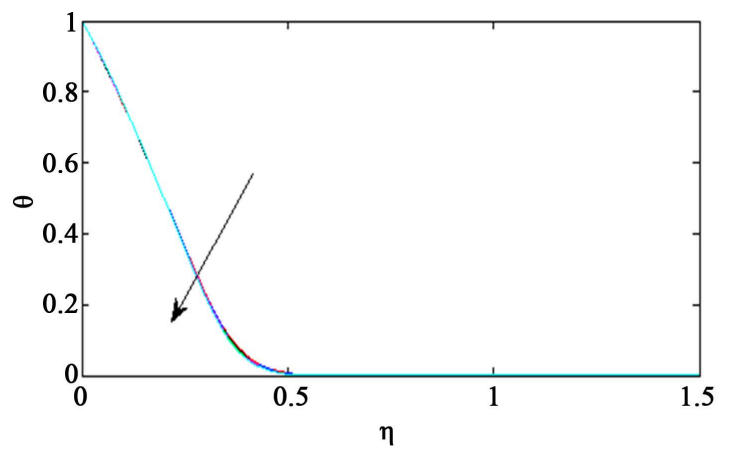

(c)

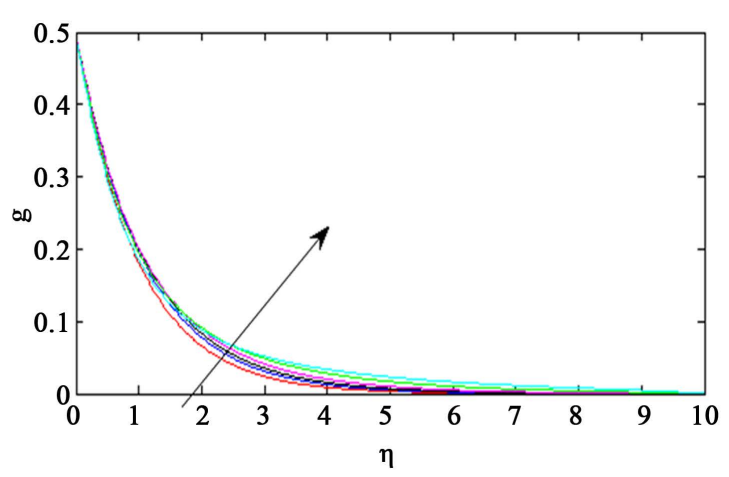

(b)

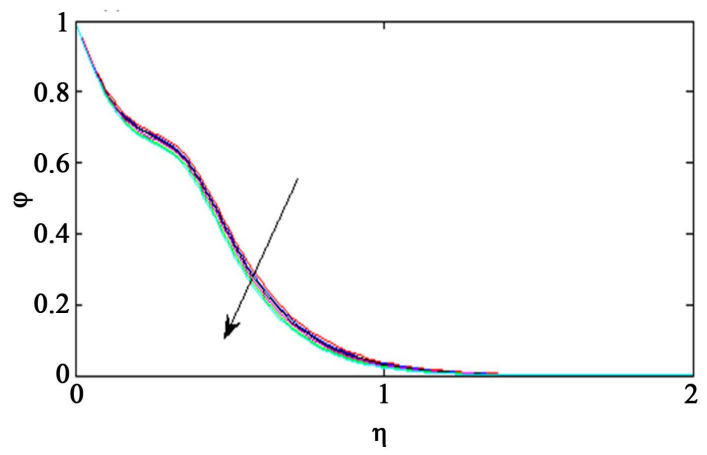

(d)

Figure 2. Effect of material parameter $(K)$ on dimensionless (a) Velocity profiles for steady flow $(\xi=1)$ at various values of $K$; (b) Angular velocity profiles for steady flow $(\xi=1)$ at various values of $K$; (c) Temperature profiles for steady flow $(\xi=1)$ at various values of $K$; (d) Profiles for steady flow $(\xi=1)$ at various values of $K$. Nanoparticle volume fraction distributions at the final steady state when $\lambda=1, \mathrm{Sc}=7, \mathrm{Pr}=100, \mathrm{Nb}=\mathrm{Nt}=\mathrm{Nr}=0.1, n=0.5$. 


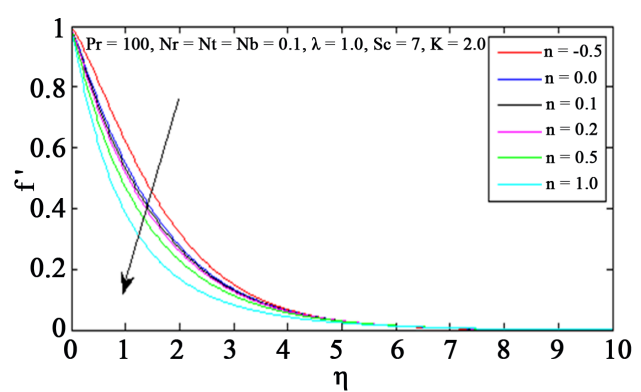

(a)

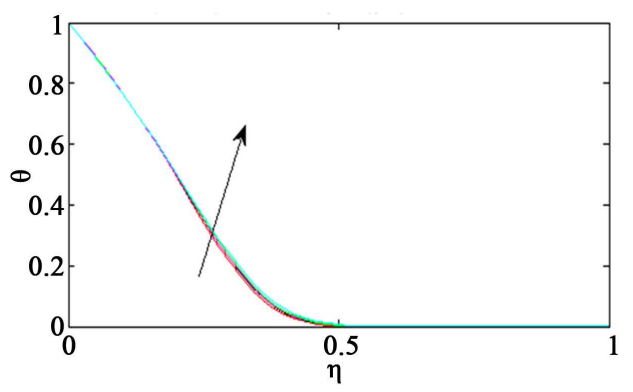

(c)

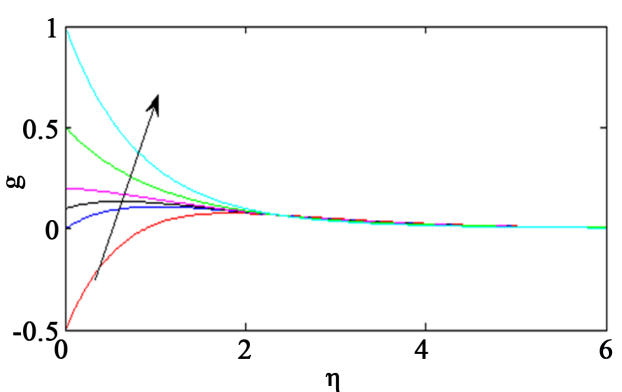

(b)

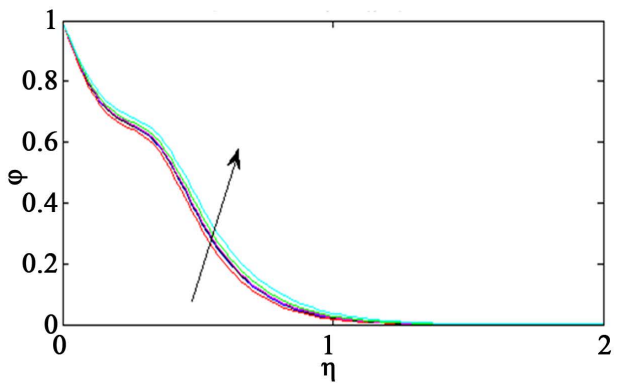

(d)

Figure 3. Effect of parameter $n$ on dimensionless (a) Velocity profiles for steady flow $(\xi=1)$ at various values of $n$; (b) Angular velocity profiles for steady flow $(\xi=1)$ at various values of $n$; (c) Temperature profiles for steady flow $(\xi=1)$ at various values of $n$; (d) Profiles for steady flow $(\xi=1)$ at various values of $n$. Nanoparticle volume fraction distributions at the final steady state when $\lambda=1, S c=7, \operatorname{Pr}=100, K=2, \mathrm{Nb}=\mathrm{Nt}=\mathrm{Nr}=0.1$.

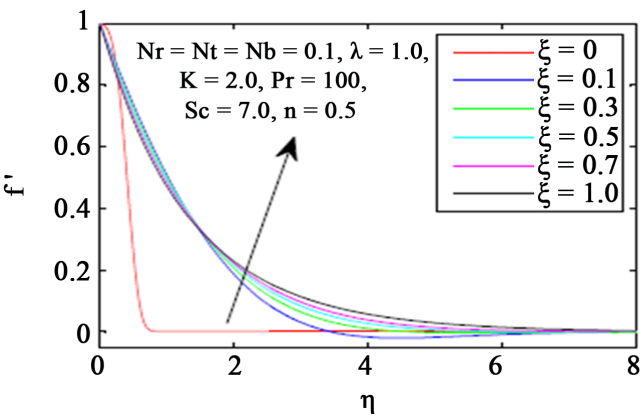

(a)

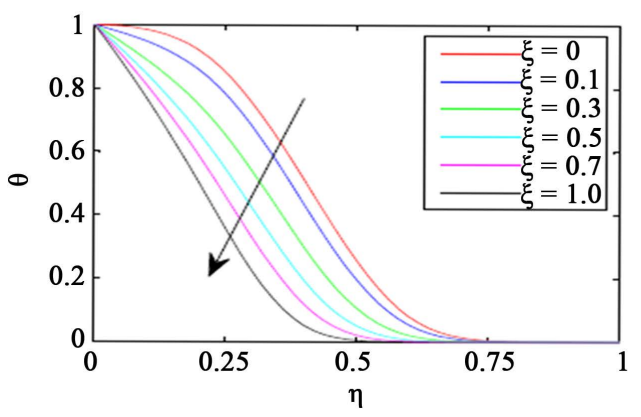

(c)

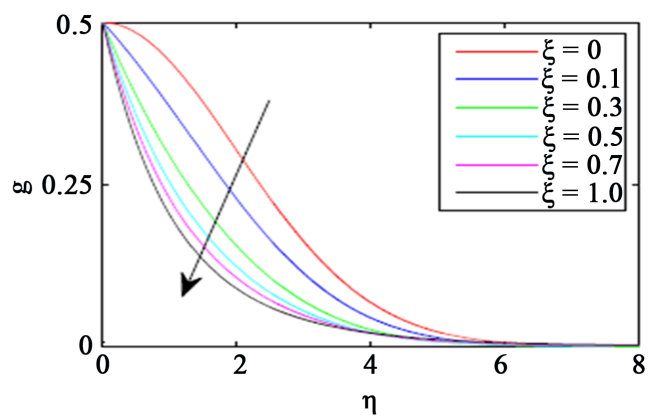

(b)

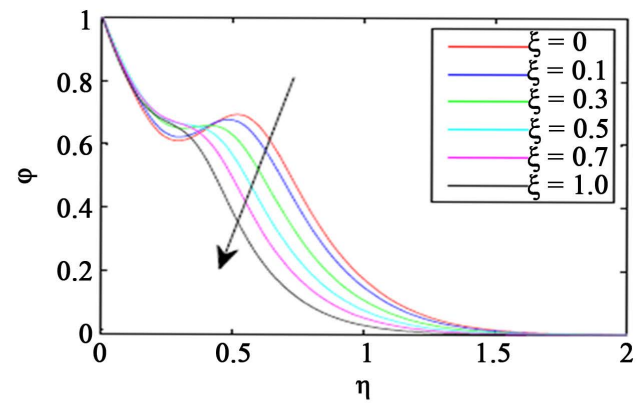

(d)

Figure 4. Unsteady fully developed flow distributions at various on dimensionless (a) Velocity profiles of early unsteady flow at various $\xi$; (b) Angular velocity profiles of early unsteady flow at various $\xi$; (c) Temperature profiles of early unsteady flow at various $\xi$; (d) Concentration profiles of early unsteady flow at various $\xi$. Nanoparticle volume fraction when $\lambda=1$, Sc $=7, \mathrm{Pr}=100, K=2, \mathrm{Nb}=\mathrm{Nt}=\mathrm{Nr}=0.1, n=0.5$. 
plication in the particular region where we specified for heat enhancement. These figures show that the velocity profiles corresponding to increasing of $\xi(0<\xi<1)$ to approach the steady profile corresponding to $\xi=1$. It can be seen that all the profiles decreases monotonically with $\eta$, and become zero for away from the surface, which also holds the verification of boundary conditions. Again it is observed that the micro-rotation, temperature and concentration profiles decreased as increasing of $\xi$ to reach steady flow.

Brownian motion is a random movement of nanoparticles and the motion occurs because of collisions with base fluid molecules. Brownian motion parameter (Nb) appears in Equations (10) and (11), Figure 5 depicts that velocity and temperature increases with increasing $\mathrm{Nb}$. The reverse trend is observed for the concentration. Brownian motion increases with an increasing of temperature. Hence the heat transfer enhancement due to collision of high thermal energy with lower energy particles. The important mechanism thermophoresis is observed when a combination of particles is subjected to a temperature gradient force. The particles move in the direction of decreasing temperature. Thermophoresis parameter (Nt) appears in Equations (10) and (11) and it plays a physically powerful role in determining the diffusion of heat and nanoparticle concentration in the boundary layer. From Figure 6, we observed that the temperature and nanoparticle concentration increase as $\mathrm{Nt}$ increases, i.e. thermal and concentration boundary layer increased so that the system would be heated.

Figure 7 shows that as buoyancy parameter $\lambda$ increases, the velocity and temperature decreased whereas micro-rotation and concentration increase. From Figure 8, the same trend is observed for Pr in the boundary layer. It is also observed that the heat transfer of the nanofluid decreases as the distance from the surface increases for small/large Pr until it reaches zero.

\section{Conclusion}

In this work, we have studied the problem of the unsteady mixed convection boundary layer flow of a non-Newtonian nanofluid over a stretching surface. Numerical results for surface heat transfer rate and mass transfer rate have been presented for parametric variations of the material parameters of the fluid, buoyancy parameter $\lambda$, Brownian motion parameter $\mathrm{Nb}$, thermophoresis parameter $\mathrm{Nt}$, Prandtl number and Schmidt number. The results indicate that as a result of the impulsive motion, the friction factor, heat transfer rate and mass trans-

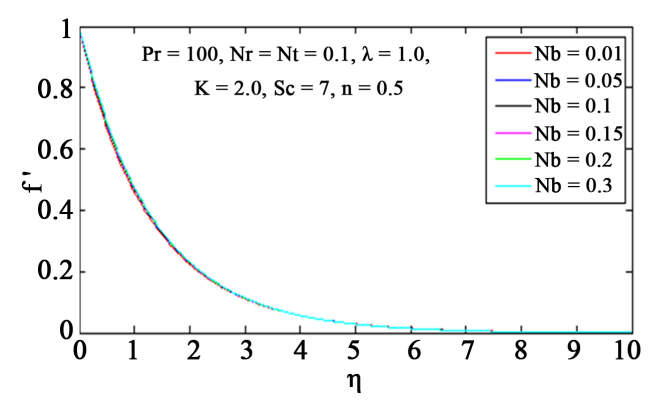

(a)

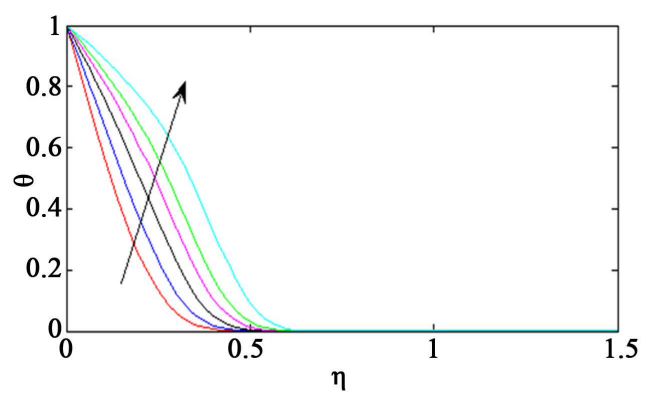

(c)

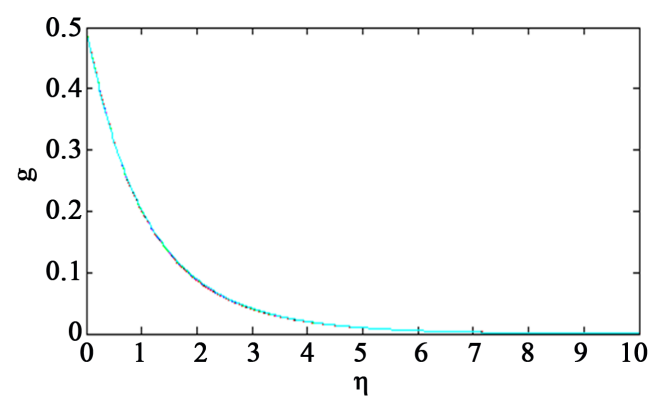

(b)

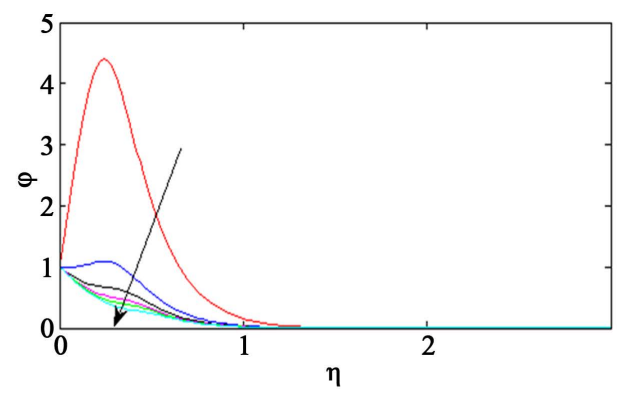

(d)

Figure 5. Effect of Brownian motion parameter $(\mathrm{Nb})$ on dimensionless (a) Velocity profiles for steady flow ( $\xi=1)$ at various values of $\mathrm{Nb}$; (b) Angular velocity profiles for steady flow $(\xi=1)$ at various values of $\mathrm{Nb}$; (c) Temperature profiles for steady flow $(\xi=1)$ at various values of $\mathrm{Nb}$; (d) Concentration profiles for steady flow $(\xi=1)$ at various values of Nb. Nanoparticle volume fraction distributions at final steady state when $\lambda=1, S c=7, \operatorname{Pr}=100, K=2, \mathrm{Nt}=\mathrm{Nr}=0.1, n=0.5$. 


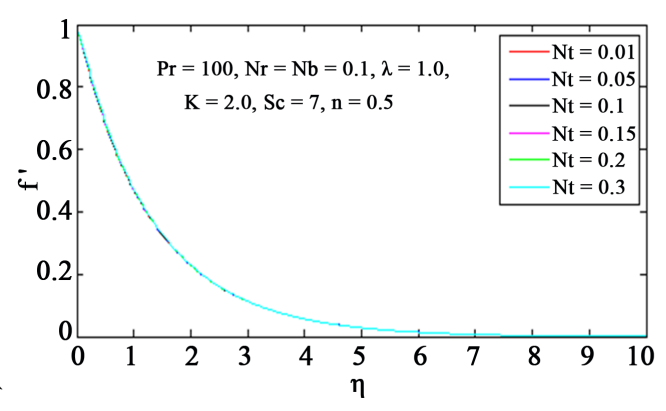

(a)

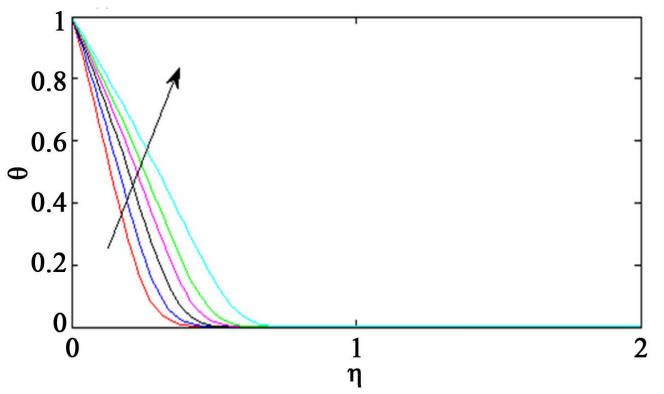

(c)

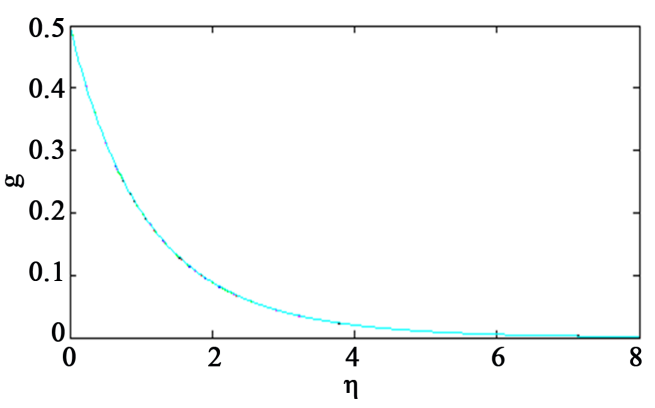

(b)

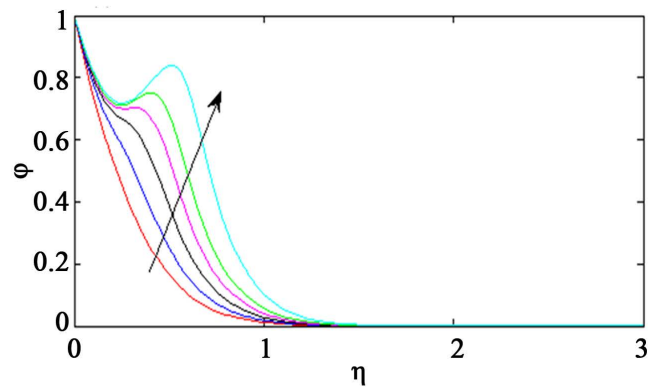

(d)

Figure 6. Effect of thermophoretic parameter (Nt) on dimensionless (a) Velocity profiles for steady flow $(\xi=1)$ at various values of Nt; (b) Angular velocity profiles for steady flow $(\xi=1)$ at various values of Nt; (c) Temperature profiles for steady flow $(\xi=1)$ at various values of Nt; (d) Concentration profiles for steady flow $(\xi=1)$ at various values of Nt. Nanoparticle volume fraction distributions at final steady state when $\lambda=1, \mathrm{Sc}=7, \operatorname{Pr}=100, K=2, \mathrm{Nb}=\mathrm{Nr}=0.1, n=0.5$.

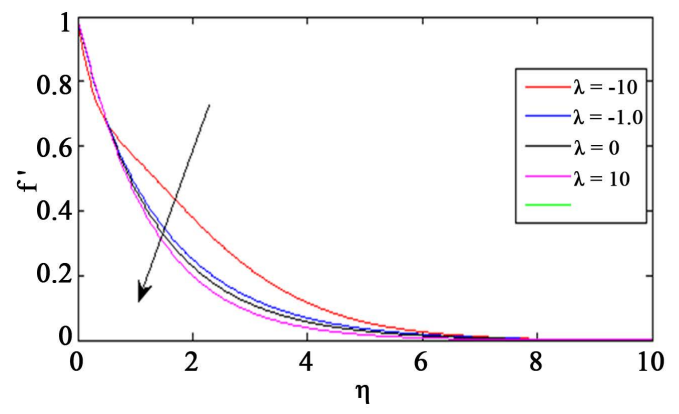

(a)

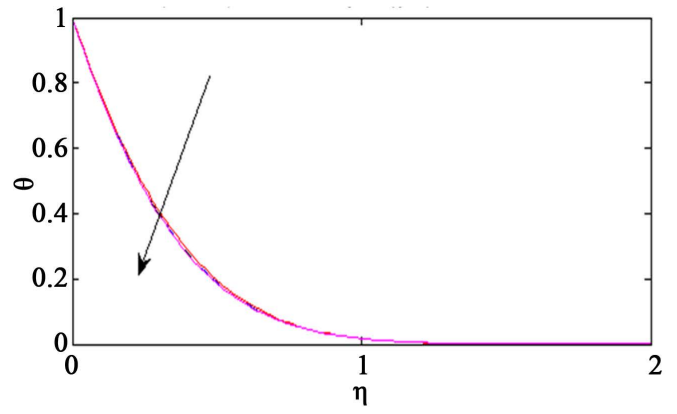

(c)

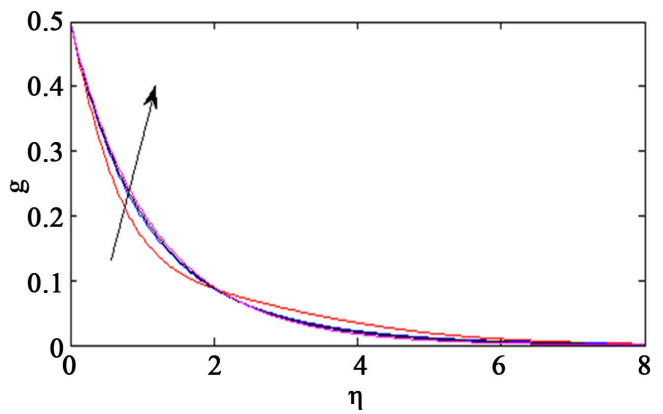

(b)

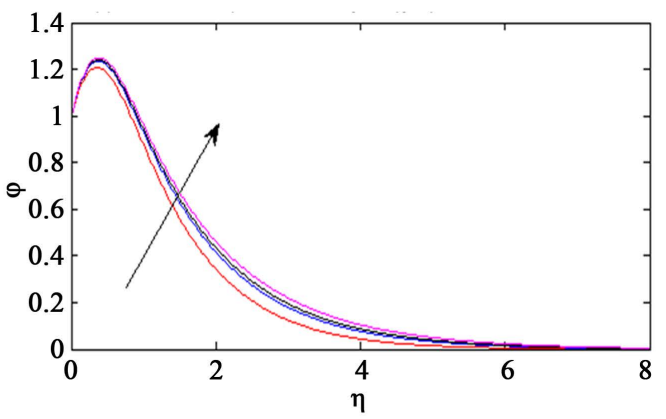

(d)

Figure 7. Effect of mixed convection parameter $(\lambda)$ on dimensionless (a) Velocity profiles for steady flow $(\xi=1)$ at various values of $\lambda$; (b) Angular velocity profiles for steady flow $(\xi=1)$ at various values of $\lambda$; (c) Temperature profiles for steady flow $(\xi=1)$ at various values of $\lambda$; (d) Concentration profiles for steady flow $(\xi=1)$ at various values of $\lambda$. Nanoparticle volume fraction distributions at final steady state when $S c=7, \operatorname{Pr}=100, K=2, \mathrm{Nb}=\mathrm{Nt}=\mathrm{Nr}=0.1, n=0.5$. 


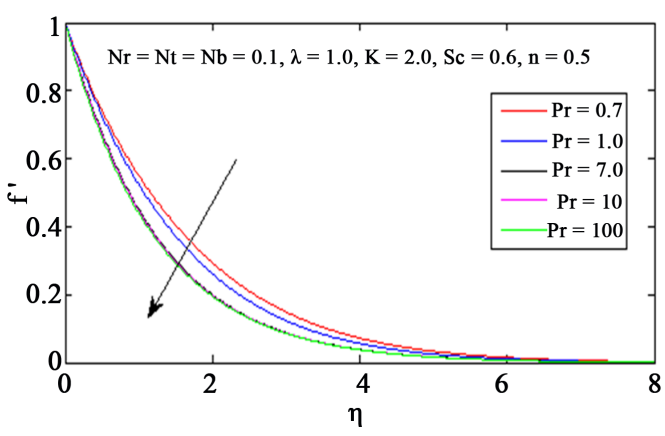

(a)

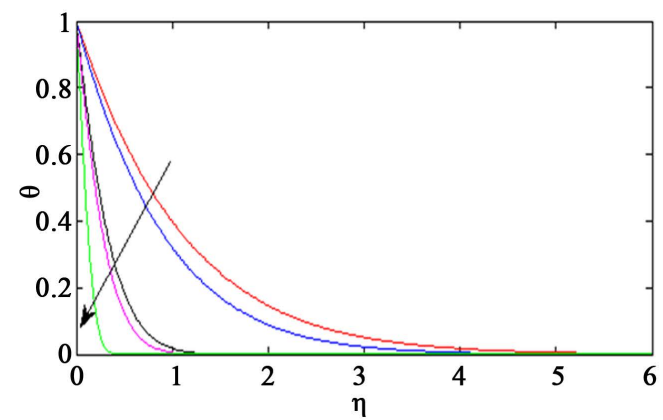

(c)

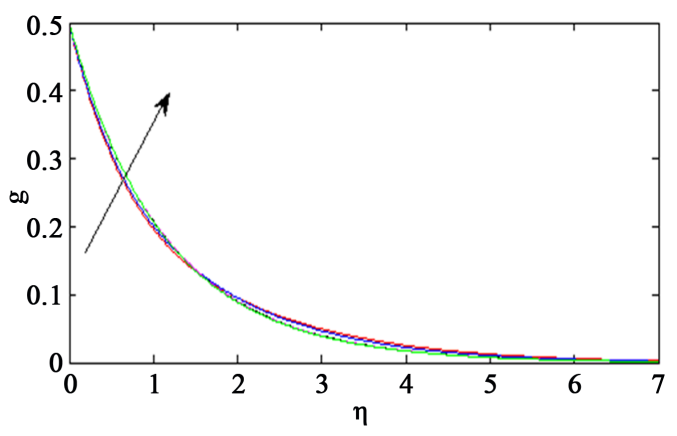

(b)

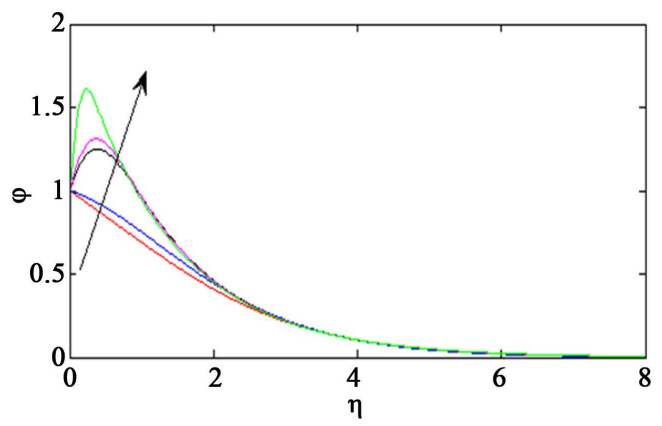

(d)

Figure 8. Effect of Prandtl number (Pr) on dimensionless (a) Velocity profiles for steady flow $(\xi=1)$ at various values of Pr; (b) Angular velocity profiles for steady flow $(\xi=1)$ at various values of Pr; (c) Temperature profiles for steady flow $(\xi=1)$ at various values of $\mathrm{Pr}$; (d) Concentration profiles for steady flow $(\xi=1)$ at various values of Pr. Nanoparticle volume fraction distributions at final steady state when $\lambda=1, \mathrm{Sc}=7, K=2, \mathrm{Nb}=\mathrm{Nt}=\mathrm{Nr}=0.1, n=0.5$.

fer rate have a larger values for small time $(\xi \approx 0)$ at the start of the motion and decrease monotonically and reach steady state at $\xi=1$. There is a smooth transition from small time (at the start of the motion) to the large time (at steady state).

The results indicate that as Nt increases, the heat transfer rate (Nusselt number) decreases whereas mass transfer rate (Sherwood number) increases. As Nb increases, the surface mass transfer rate increases whereas the surface heat transfer rate decreases.

\section{References}

[1] Das, S.K., Choi, S.U.S., Yu, W. and Pradet, T. (2007) Nanofluids: Science and Technology. Wiley, Hoboken. http://dx.doi.org/10.1002/9780470180693

[2] Buongiorno, J. (2006) Convective Transport in Nanofluids. Journal of Heat Transfer, 128, 240-250. http://dx.doi.org/10.1115/1.2150834

[3] Xuan, Y.M. and Roetzel, W. (2000) Conceptions for Heat Transfer Correlation of Nanofluids. International Journal of Heat and Mass Transfer, 43, 3701-3707. http://dx.doi.org/10.1016/S0017-9310(99)00369-5

[4] Yu, W. and Xie, H.Q. (2011) A Review on Nanofluids: Preparation, Stability Mechanisms, and Applications, Hindawi Publishing Corporation. Journal of Nanomaterials, 2012, Article ID: 435873.

[5] Mahbubul, I.M., Saidur, R. and Amalina, M.A. (2012) Latest Developments on the Viscosity of Nanofluids. International Journal of Heat and Mass Transfer, 55, 874-885. http://dx.doi.org/10.1016/j.ijheatmasstransfer.2011.10.021

[6] Aberoumand, S., Aberoumand, H. and Javaherdeh, K. (2013) Improve Heat Transfer by Using Nanofluids: A Review. American Journal of Advanced Scientific Research (AJASR), 1, 375-386.

[7] Gupta, M., Arora, N., Kumar, R., Kumar, S. and Dilbaghi, N. (2014) A Comprehensive Review of Experimental Investigations of Forced Convective Heat Transfer Characteristics for Various Nanofluids. International Journal of Mechanical and Materials Engineering, 9, 1-21. http://dx.doi.org/10.1007/s11242-009-9413-2

[8] Kuznetsov, A.V. and Nield, D.A. (2010) Thermal Instability in a Porous Medium Layer Saturated by a Nanofluid: Brinkman Model. Transport in Porous Media, 81, 409-422. http://dx.doi.org/10.1007/s11242-009-9413-2 
[9] Kuznetsov, A.V. and Nield, D.A. (2011) Double-Diffusive Natural Convective Boundary-Layer Flow of a Nanofluid past a Vertical Plate. International Journal of Thermal Sciences, 50, 712-717. http://dx.doi.org/10.1016/j.ijthermalsci.2011.01.003

[10] Bachok, N., Ishak, A. and Pop, I. (2012) Flow and Heat Transfer Characteristics on a Moving Plate in a Nanofluid. International Journal of Heat and Mass Transfer, 55, 642-648. http://dx.doi.org/10.1016/j.ijheatmasstransfer.2011.10.047

[11] Gorla, R.S.R., Chamkha, A.J. and Hossain, A. (2009) Mixed Convection Flow of Non-Newtonian Fluid from a Slotted Vertical Surface with Uniform Surface Heat Flux. Canadian Journal of Chemical Engineering, 87, 534-540. http://dx.doi.org/10.1002/cjce.20195

[12] Gorla, R.S.R. and Kumari, M. (2013) Mixed Convection in an Axisymmetric Stagnation Flow of a Non-Newtonian Nanofluid on a Vertical Cylinder. Proceedings of the Institution of Mechanical Engineers, Part N: Journal of Nanoengineering and Nanosystems, 227, 150-160.

[13] Chamkha, A.J., Rashad, A.M. and Aly, A. (2013) Transient Natural Convection Flow of a Nanofluid over a Vertical Cylinder. Meccanica, 48, 71-81. http://dx.doi.org/10.1007/s11012-012-9584-8

[14] RamReddy, Ch., Murthy, P.V.S.N., Chamkha, A.J. and Rashadd, A.M. (2013) Soret Effect on Mixed Convection Flow in a Nanofluid under Convective Boundary Condition. International Journal of Heat and Mass Transfer, 64, 384-392. http://dx.doi.org/10.1016/j.ijheatmasstransfer.2013.04.032

[15] Vendabai, K. and Sarojamma, G. (2014) Unsteady Convective Boundary Layer Flow of a Nanofluid over a Stretching Surface in the Presence of a Magnetic Field and Heat Generation. International Journal of Emerging Trends in Engineering and Development, 3, 214-230.

[16] Kumari, M., Gireesha, B.J. and Gorla, R.S.R. (2015) Heat and Mass Transfer in a Nanofluid Film on an Unsteady Stretching Surface. Journal of Nanofluids, 4, 560-567.

[17] Eringen, A.C. (1966) Theory of Micropolar Fluids. Journal of Mathematics and Mechanics, 16, 1-18. http://dx.doi.org/10.1512/iumj.1967.16.16001

[18] Eringen, A.C. (2001) Microcontinuum Field Theories II: Fluent Media. Springer, New York.

[19] Ariman, T., Turk, M.A. and Sylvester, N.D. (1973) Microcontinuum Fluid Mechanics-A Review. International Journal of Engineering Science, 11, 905-930. http://dx.doi.org/10.1016/0020-7225(73)90038-4

[20] Ariman, T., Turk, M.A. and Sylvester, N.D. (1974) Applications of Micro-Continuum Fluid Mechanics. International Journal of Engineering Science, 12, 273-293. http://dx.doi.org/10.1016/0020-7225(74)90059-7

[21] Bég, O.A., Bhargava, R., Rawat, S., Takhar, H.S. and Bég, T.A. (2007) Numerical Study of Grashof and Darcy Number Effects on Natural Convection Heat and Species Transfer Past a Stretching Surface in Micropolar Saturated-Porous Medium with Viscous Heating. International Journal of Fluid Mechanics Research, 34, 287-307. http://dx.doi.org/10.1615/InterJFluidMechRes.v34.i4.10

[22] Kim, Y.-J. (2004) Heat and Mass Transfer in MHD Micropolar Flow over a Vertical Moving Porous Plate in a Porous medium. Transport in Porous Media, 56, 17-37. http://dx.doi.org/10.1023/B:TIPM.0000018420.72016.9d

[23] Gorla, R.S.R., Slaouti, A. and Takhar, H.S. (1998) Free Convection in Micropolar Fluids over a Uniformly Heated Vertical Plate. International Journal of Numerical Methods for Heat \& Fluid Flow, 8, 504-518. http://dx.doi.org/10.1108/09615539810220261

[24] Rees, D.A.S. and Bassom, A.P. (1996) The Blasius Boundary Layer Flow of a Micropolar Fluid. International Journal of Engineering Science, 34, 133-124. http://dx.doi.org/10.1016/0020-7225(95)00058-5

[25] Lien, F.S. and Chen, C.C. (1987) Mixed Convection of Micropolar Fluid about a Sphere with Blowing and Suction. International Journal of Engineering Science, 34, 1301-1310.

[26] Nazar, R., Amin, N., Grosan, T. and Pop, I. (2003) Mixed Convection Boundary Layer Flow about an Isothermal Sphere in Micropolar Fluid. International Journal of Thermal Sciences, 42, 283-293. http://dx.doi.org/10.1016/S1290-0729(02)00027-3

[27] Kumari, M., Slaouti, A., Takhar, H.S., Nakamura, S. and Nath, G. (1996) Unsteady Free Convection Flow over a Continuous Moving Vertical Surface. Acta Mechanica, 116, 75-82. http://dx.doi.org/10.1007/BF01171421

[28] Ishak, A., Nazar, R. and Pop, I. (2006) Unsteady Mixed Convection Boundary Layer Flow Due to a Stretching Vertical Surface. The Arabian Journal of Science and Engineering, 31, 165-182.

[29] Ishak, A., Nazar, R. and Pop, I. (2008) Heat Transfer over a Stretching Surface with Variable Surface Heat Flux in Micropolar Fluids. Physics Letters A, 372, 559-561. http://dx.doi.org/10.1016/j.physleta.2007.08.003

[30] Pop, I. and Na, T.Y. (1996) Unsteady Flow Past a Stretching Sheet. Mechanics Research Communications, 23, 413422. http://dx.doi.org/10.1016/0093-6413(96)00040-7

[31] Wang, C.Y., Du, G., Miklavcic, M. and Chang, C.C. (1997) Impulsive Stretching of a Surface in a Viscous Fluid. 
SIAM (Society for Industrial and Applied Mathematics) Journal on Applied Mathematics, 57, 1-14. http://dx.doi.org/10.1137/S0036139995282050

[32] Rashad, A.M. and EL-Kabeir, S.M.M. (2010) Heat and Mass Transfer in Transient Flow by Mixed Convection Boundary Layer over a Stretching Sheet Embedded in a Porous Medium with Chemically Reactive Species. Journal of Porous Media, 13, 75-85. http://dx.doi.org/10.1615/JPorMedia.v13.i1.70

[33] Rohni, A.M., Ahmad, S. and Pop, I. (2012) Flow and Heat Transfer over an Unsteady Shrinking Sheet with Suction in Nanofluids. International Journal of Heat and Mass Transfer, 55, 1888-1895. http://dx.doi.org/10.1016/j.ijheatmasstransfer.2011.11.042

[34] Cebeci, T. and Bradshaw, P. (1984) Physical and Computational Aspects of Convective Heat Transfer. Springer, New York. http://dx.doi.org/10.1007/978-3-662-02411-9

[35] Keller, H.B. (1978) Numerical Methods in Boundary-Layer Theory. Annual Review of Fluid Mechanics, 10, 417-433. http://dx.doi.org/10.1146/annurev.fl.10.010178.002221

[36] Vasu, B., Prasad, V.R., Bég, O.A., Aziz, A. and Prashad, R.D. (2010) Numerical Analysis of Magnetohydrodynamic Nonlinear Convection Heat and Mass Transfer from a Sphere in a Non-Darcian Variable-Porosity Medium. International Journal of Applied Mathematics, 17, 64-111. 